\title{
"Our Hands Are Tied Until Your Doctor Gets Here": Nursing Perspectives on Inter-hospital Transfers
}

\author{
Amy Yu, MD ${ }^{7}$, Sarah R. Jordan, $M A^{2}$, Heather Gilmartin, $P h D, N P^{3,4}$, \\ Stephanie k. Mueller, MD, MPH ${ }^{5}$, Brooke Dorsey Holliman, PhD', and Christine D. Jones, \\ $M D, M S^{1,3}$
}

\begin{abstract}
'Division of Hospital Medicine, Department of Medicine, University of Colorado Anschutz Medical Campus, 12401 E. 17th Avenue Mailstop F-782, Aurora, CO, USA; 'Division of Geriatric Medicine, Department of Medicine, University of Colorado Anschutz Medical Campus, Aurora, CO, USA; ${ }^{3}$ Veterans Health Administration, Eastern Colorado Health Care System, Denver-Seattle Center of Innovation for Veteran-Centered and Value Driven Care, Aurora, CO, USA; ${ }^{4}$ Department of Health Systems, Management and Policy, Colorado School of Public Health, University of Colorado Anschutz Medical Campus, Aurora, CO, USA; ${ }^{5}$ Division of General Internal Medicine, Brigham and Women's Hospital, Boston, MA, USA;

${ }^{6}$ Department of Family Medicine, University of Colorado Denver School of Medicine, Aurora, CO, USA.
\end{abstract}

BACKGROUND: The transfer of patients between hospitals (inter-hospital transfer, or IHT) is a common occurrence for patients, but guidelines to ensure safe and effective IHTs are lacking. Poor IHTs result in higher rates of mortality, longer lengths of stay, and higher hospitalization costs compared to admissions from the emergency department. Nurses are often the first point of contact for IHT patients and can provide valuable insights on key challenges to IHT processes.

OBJECTIVE: To characterize the experiences of inpatient floor-level bedside nurses caring for IHT patients and identify care coordination challenges and solutions.

DESIGN/PARTICIPANTS/APPROACH: Qualitative study using semi-structured focus groups and interviews conducted from October 2019 to July 2020 with 21 inpatient floor-level nurses caring for adult medicine patients at an academic hospital. Nurses were recruited using a purposive convenience sampling approach. A combined inductive and deductive coding approach guided by thematic analysis was used for data analysis.

KEY RESULTS: Results from this study are mapped to the Agency for Healthcare Research and Quality Care Coordination Measurement Framework domains of communication, assessing needs and goals, and negotiating accountability. The following key themes characterize nurses' experiences with IHT related to these domains: (1) challenges with information exchange and team communication during IHT, (2) environmental and information preparation needed to anticipate transfers, and (3) determining responsibility and care plans after the IHT patient has arrived at the accepting facility.

CONCLUSIONS: Nurses described the absence of standardized processes to coordinate care before or at the time of patient arrival. Challenges to communication and coordination during IHTs negatively impacted patient care

Prior Presentations:

1. Society of General Internal Medicine 2020 New England \& Mountain West Virtual Regional Conference, Poster Presentation, Nov 2020

2. Society of General Internal Medicine 2021 Annual Conference, Poster Presentation, April 2021

Received May 30, 2021

Accepted November 10, 2021

Published online January 6, 2022 and nursing professional satisfaction. To streamline care for IHT patients and reduce nursing stress, future IHT interventions should include standardized handoff reports, timely identification and easy access to admitting clinicians, and timely clinician evaluation and orders.

KEY WORDS: interhospital transfer; inter-hospital transfer; qualitative; nursing; care transitions.

J Gen Intern Med 37(7):1729-36

DOI: $10.1007 / \mathrm{s} 11606-021-07276-5$

(C) The Author(s) under exclusive licence to Society of General Internal Medicine 2021

\section{INTRODUCTION}

The transfer of patients between hospitals (inter-hospital transfer, or IHT) is common, affecting over one million patients each year in the USA. ${ }^{1-3}$ Notably, IHT involves approximately $10 \%$ of Medicare admissions and $3.5 \%$ of all hospital inpatient admissions. ${ }^{1-3}$ Despite the frequency of IHTs, national guidelines are not available to guide clinical processes or define benchmarks to assess care across facilities. ${ }^{4,5}$ Only recommendations regarding the expertise and equipment required for IHT transport and disease-specific targets (e.g., door to balloon time for ST segment elevation acute myocardial infarction) are available. ${ }^{6-9}$ This gap in general guidance forces healthcare organizations to create their own IHT protocols, introducing inconsistencies in care coordination of a multi-step, multi-person process (Fig. 1).

Care coordination is defined by the Agency for Healthcare Research and Quality (AHRQ) as "the deliberate organization of patient care activities between two or more participants involved in a patient's care to facilitate the appropriate delivery of health care services." ${ }^{10}$ Organization of care involves the alignment of appropriate personnel and resources and is directed by information exchange. ${ }^{10}$ The variability in IHT processes across institutions and the services within them can lead to fragmented communication structures and ambiguous care roles. ${ }^{2,11-13}$ Existing data in other areas of care 


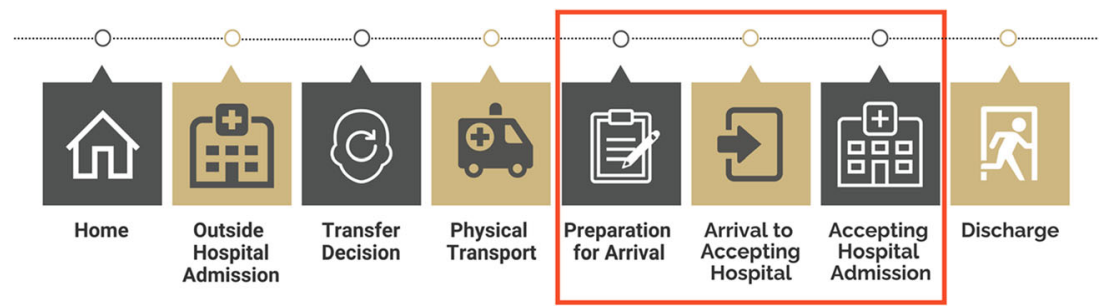

Figure 1 IHT process overview. The inter-hospital transfer (IHT) process is a multi-step process with multiple participants. Our study focuses on the nursing experience during the areas of transitions enclosed in the box.

transitions, such as the transition from inpatient to outpatient settings, establish that inadequate care coordination plays a role in poor patient outcomes ${ }^{14-18}$ and is therefore a critical area in IHT to study.

Nurses frequently are the first clinicians to interact with patients upon arrival at the accepting facility; thus, nurses' perspectives are integral to better characterizing the IHT process and identifying potential areas for targeted interventions. Prior literature has primarily explored only the patient and physician perspectives $2,4,11-13,19$ or focused on nursing perspectives during the transport of critically ill patients. ${ }^{20,21}$

This study adds to existing literature by focusing on the experiences of inpatient floor-level bedside nurses at accepting facilities. Their experiences, which span from preparing for IHT patients prior to arrival to admitting IHT patients at the accepting facility (bracketed in Fig. 1), occur when timely and accurate coordination of care between healthcare providers is essential for safe and streamlined patient care. Bedside nurses can best highlight coordination gaps since they are not involved in the transfer decision (in contrast to critical care nurses who staff call centers) and must coordinate between multiple clinicians. Additionally, floor-level IHT patients have greater variability in arrival times compared to critically ill patients whose transport is often expedited, adding a level of complexity to floor-level nursing care. Our primary goal is to identify challenges and solutions to IHT care coordination from the perspective of nurses to guide interventions to standardize the IHT process.

\section{METHODS}

\section{Design and Setting}

This is a qualitative descriptive study of inpatient floor-level nursing experiences with IHT at a quaternary care academic medical center in Colorado. This hospital receives over 5000 IHTs annually from emergency rooms and acute care hospitals across the Rocky Mountain and Southwest regions of the USA. Floor-level medicine patients comprise the largest percentage of IHT patients (44\%) with surgical patients $(36 \%)$ following. IHTs are managed by the health system's call center, which is staffed by critical care trained nurses who receive transfer request calls and coordinate clinician contact, transportation logistics, and bed assignments. Floor-level bedside nurses are responsible for preparing for IHT patient arrival and coordinating care once the IHT patient arrives.

\section{Participants}

We used a purposive convenience sampling strategy ${ }^{22-24}$ to recruit inpatient floor-level nurses from units with the highest volumes of medicine IHTs and who had experience working with IHT patients. First, we contacted nursing leadership on primarily medicine-focused units via email or in-person to establish interest. Unit leadership then granted permission to recruit staff for this study via email, in-person at unit meetings, and/or by word of mouth. Nurses had the option to participate in individual interviews or focus groups per their availability. We emphasized to participants that their participation was not tied to their clinical role.

\section{Data Collection}

Interviews (one participant) and focus groups (two or more participants) were held over 10 months from October 2019 to July 2020. Focus groups and interviews lasted up to $1 \mathrm{~h}$ and were both conducted in-person (pre-COVID-19) and by telephone. Two interviewers facilitated focus groups and interviews, one who had a pre-existing professional relationship to participants as a physician (AY) and one who did not have any pre-existing relationship to participants and had experience conducting qualitative data collection (SRJ). ${ }^{25}$ Both interviewers conducted in-person focus groups together and conducted in-person interviews, telephone interviews, and telephone focus groups individually.

A semi-structured interview guide (Appendix 1) was used to explore nursing experiences in IHT care and challenges and solutions to successful IHT. The AHRQ Care Coordination Measurement Framework ${ }^{10}$ (Appendix 2) was used to provide a conceptual model that informed interview guide development and data analysis. This framework organizes measures of care coordination into specific activities or broad approaches, including domains of accountability, communication, and assessment of needs and goals. These domains offer a lens to examine IHT care coordination activities specific to the nursing perspective. Given the emergence of COVID-19 during the study period, questions were added halfway through the study to examine the impact of COVID-19 on nurses' experience with IHTs. 
Interviews and focus groups were audio recorded, professionally transcribed, and de-identified. Data collection occurred until theoretical data saturation was reached, when additional data did not lead to any new codes or emergent themes. $^{26-28}$ This study was reviewed and deemed exempt by the Colorado Multiple Institutional Review Board and informed consent was obtained.

\section{Data Analysis}

Guided by thematic analysis, we used a combined inductive and deductive approach allowing for discovery of unexpected themes and categorization with the AHRQ Care Coordination Measurement Framework. We analyzed a set of transcripts individually and designed a code book together (AY, SRJ). The codebook was refined as new patterns emerged. ${ }^{27,29,30}$ Each transcript was coded in ATLAS.ti (Version 8, Berlin, Germany) by at least two coders (AY and SRJ), with $41 \%$ of the data double-coded for enhanced reliability. We maintained a record of all analytical decisions and team discussions. We established consensus by identifying and resolving differences in emerging themes through discussion and triangulation with team members (AY, SRJ, CDJ).

\section{RESULTS}

Of 200 nurses invited to participate across four large units, 21 participated (response rate 10.5\%) in a total of three focus groups and fourteen interviews. Nineteen nurses worked on adult general or subspecialty medicine units, and two nurses worked on surgical-focused floors but had overlap taking care of medicine patients. Sixteen $(76 \%)$ participants worked the day shift, three (14\%) worked night shifts, and two (10\%) worked both shifts. Seventeen $(81 \%)$ participants identified as female. Average years of working as a nurse among participants ranged from 1 to 21 years (mean 5.4 years). Demographic data are displayed in Table 1.

Results from this study are mapped to the AHRQ Care Coordination Measurement Framework domains of

Table 1 Self-Reported Demographics of Interview Sample $(N=21)$

\begin{tabular}{ll}
\hline \hline Demographic characteristic & $\begin{array}{l}\text { Nurses } \\
\boldsymbol{n}(\%)\end{array}$ \\
\hline Female & $17(81)$ \\
Charge nurse & $6(29)$ \\
Average years total working in nursing, years (SD) & 5.4 \\
& $(5.0)$ \\
Average years total on General Medicine Inpatient Ward, & $4(3.2)$ \\
years (SD) & 3.7 \\
Average years total working at hospital site, years (SD) & $(3.1)$ \\
Shift & $16(76)$ \\
$\quad$ Day shift only & $3(14)$ \\
$\quad$ Night shift only & $2(10)$ \\
Both day and night shifts & $10(48)$ \\
Unit General internal medicine & $9(43)$ \\
$\quad$ Medicine subspecialties & $2(9)$ \\
\hline Surgical specialties &
\end{tabular}

communication, assessing needs and goals, and negotiating accountability. ${ }^{10}$ The following key themes elucidate nurses' IHT experiences related to these domains: (1) challenges with information exchange and team communication during IHT, (2) environmental and information preparation needed to anticipate transfers, and (3) determining responsibility and care plans after the IHT patient arrived at the accepting facility. Findings are summarized in Table 2, and themes are presented as challenges and solutions participants identified within each domain.

\section{THEME 1. COMMUNICATION DURING IHT}

In theme 1, nurses described their experiences navigating varying levels of information and communication processes during IHT.

\section{1a. Information Exchange During Nursing Handoff Report.}

Nurses described that IHT patient care started with a nurse handoff report given over the phone by the transferring hospital's nurse. This verbal report served as the main source of information about IHT patients due to inconsistent access to a shared electronic health record. Incomplete or inaccurate handoff reports made it difficult for nurses to accurately assess the patient's clinical acuity and needs, creating uncertainty about what to expect:

"What we know about the patient is always kind of up in the air... sometimes what we get is very different than what we heard about and sometimes what we get is exactly what we heard about. It's kind of a guessing game when the patient arrives what we're actually going to be dealing with." (Interview 3)

Many participants relayed that variability in nursing experience, specialty focus, and/or acuity level of the transferring hospital influenced the quality and content of the report:

"It's harder from outside facilities knowing if you can actually trust what they're telling you... sometimes the language that we speak is a lot different...[and] the priorities are different too." (Focus group 8)

Given such broad variability, suggested solutions included standardizing some components in the handoff report, while allowing for some flexibility:

"I think if there was a streamlined bullet point report... [and] there were maybe five items that we cover and those are the core things that we need to know... I think [those core items] would definitely change a little bit depending on where the patient was going or what type of patient they are." (Interview 10) 
Table 2 AHRQ Care Coordination Domains and IHT Challenges and Solutions

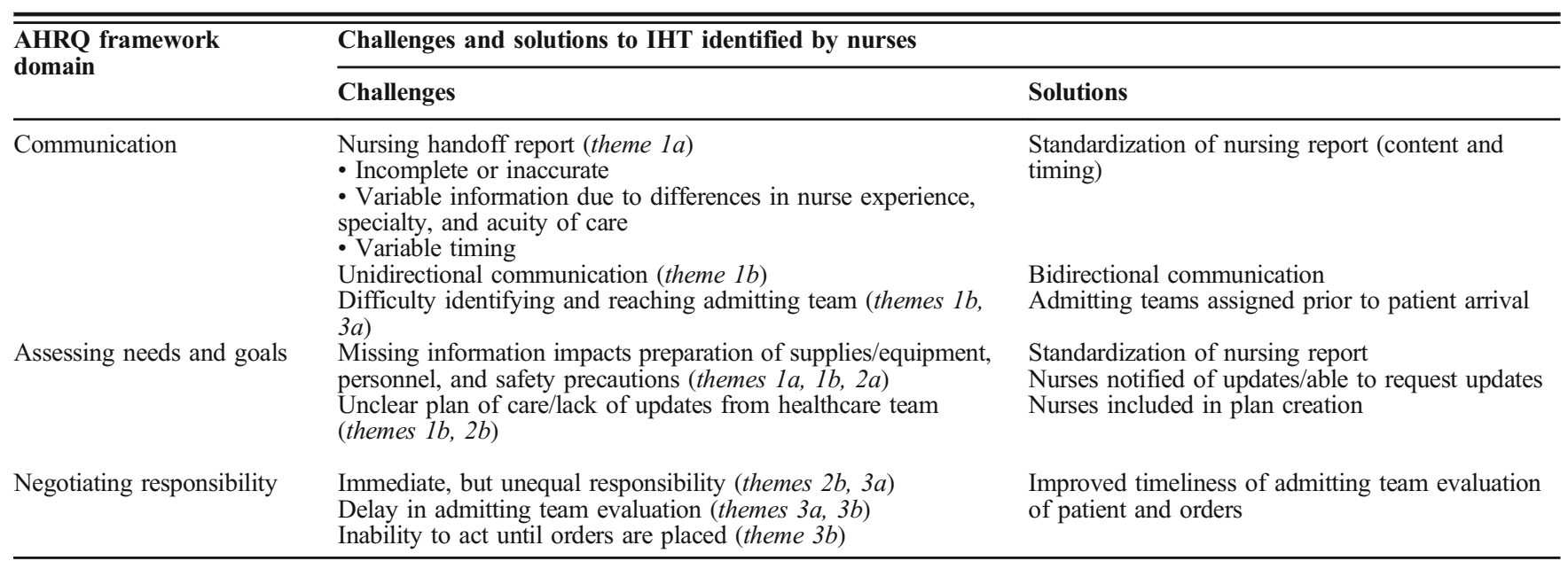

Theme 1: communication during IHT; 1a: information exchange during nursing handoff report, $1 b$ : communication structures with transferring and admitting teams; theme 2: environmental and information preparation for IHT; 2a: environmental preparation, 2b: informational preparation; theme 3: negotiating responsibility of patient care during IHT; $3 a$ : assumption of responsibility, $3 b$ : resumption of care

AHRQ Agency for Healthcare Research and Quality, IHT inter-hospital transfer

Additional issues that influenced fidelity of information exchange included timing of the report, which ranged from hours before a patient arrived to after the patient arrived, as well as timing of patient arrival in relation to nursing shift changes.

\section{1b. Communication Structures with Transferring and} Admitting Teams. Nurses described communication structures to facilitate information exchange as unidirectional, "like a one-way communication" (Focus group 4), with nurses primarily identifying as recipients.

Firstly, when receiving the nursing handoff report, nurses described having to take the report whenever the call center contacted them:

"Between the surprise element of not knowing when [the call center] is going to call and then having to take that call when they do call... it's kind of stressful." (Interview 15)

Secondly, most participants relayed that there was no process for receiving updates about changes in patient's clinical status during transport. They described only learning about changes if the transferring nurse contacted the call center or from transport personnel at time of patient arrival.

Thirdly, once IHT patients arrived at the accepting hospital, nurses voiced difficulty in communicating with the admitting team because teams were not assigned until IHT patient arrival and admitting teams did not regularly initiate communication with nurses. Many nurses described paging different teams until the right provider was found:
"They're like, "Okay, well, try this person" and it's just like a never ending "call him" and "call him."” (Interview 17)

A solution most participants suggested involved assignment of an admitting team prior to patient arrival:

"I just feel like if someone is in the hospital, they should have a provider assigned to them from the getgo. That way, we know who to call." (Interview 14)

Lastly, nurses described not being consistently apprised of the patient's care plan by admitting providers, often discovering the plan by inferring from orders, reading provider documentation, and/or asking patients:

"It's like I'm out of the loop. I don't know what's happening and I don't know what's going on. Sometimes, I ask the patients, 'What did the doctor tell you?' Which is kind of odd... I should know because the doctors and nurses should be communicating." (Interview 13)

Participants identified closed-loop, timely, and bidirectional communication as integral to successful information exchange. This was most often described during reflections on admitting team and nurse communication:

"I think it would be great if the nurse and the provider, once the patient is admitted and they kind of have a plan going forward, if they could have it established somewhere like, 'Hey, this is what the plan is. What are your concerns? These are my concerns.' Just have a 
conversation about that so everyone is on the same page." (Interview 14)

\section{THEME 2. ENVIRONMENTAL AND INFORMATIONAL PREPARATION FOR IHT}

In theme 2 , nurses reported factors that impacted their ability to set up the patients' rooms for optimal care delivery and manage IHT patients' care plan expectations.

2a. Environmental Preparation. Nurses described that key information missing from nursing handoff impaired their ability to anticipate necessary supplies and equipment (e.g., oxygen tubing or telemetry boxes), appropriate personnel (e.g., to move a patient from stretcher to bed), and/or safety precautions (e.g., personal protective equipment):

"If you don't know that a patient is going to be on contact precautions or anything like that, how many people have been in and out of that room at that point, but they haven't been wearing proper protective gear... you want to make sure that you're creating a safe environment for your patients to be in from the moment they get there." (Interview 12)

As detailed in theme 1, interviewees expressed support for some standardization of the handoff report and bidirectional communication. Comprehensive information about patient conditions and clinical change updates were identified as essential to ensure appropriate utilization of resources for patient care and safety.

2b. Informational Preparation. As the first point of contact for IHT patients at time of arrival to the accepting facility, nurses expressed the expectations that they serve as the healthcare team spokesperson and are prepared to answer patient questions. One issue that made this difficult for nurses was not understanding why certain IHT patients were transferred:

"I always ask why they're [IHT patients] coming here, which sometimes, I may never get an answer." (Interview 13)

Additionally, having to wait until IHT patients arrive to have a team assigned and not being apprised of the care plan (described above) made it difficult to appear prepared:

"[IHT patients] expect us to be expecting them, and we almost seem unprepared because... we don't know who the doctor is right away, what the plan is, or what any of the rest of the orders are." (Interview 16)

Several participants emphasized that understanding the plan before the IHT patient's arrival allowed for a streamlined transition:

"It's just good to know the plan because if you know that ahead of time, you're ready to go and you can prepare... and then things will go much smoother." (Focus group 8)

\section{THEME 3: NEGOTIATING RESPONSIBILITY OF PATIENT CARE DURING IHT}

In theme 3 , nurses discussed the extent of their patient care responsibilities and their limited ability to act on those responsibilities until provider orders were available.

3a. Assumption of Responsibility. Nurses recounted assuming immediate responsibility for IHT patients' well-being and needs once they arrived. Nurses described that their physical presence and accessibility, combined with the unknown timing of admitting team evaluation, made them naturally accountable for the patient:

"We take on the responsibility of caring for that patient immediately, and while the doctor is important obviously for orders and stuff like that, we're really the ones that are taking care of that patient for probably the first good three to four hours depending on what the admitting team's workflow looks like." (Interview 3)

Several emphasized nursing's critical role in providing the first evaluation of the patient's clinical stability and immediate care needs at time of patient arrival:

"These doctors that we're talking to... they've never seen this patient before. They might have not even read a single word about them, so it really comes down heavy on nursing to be aware of the patient's condition when they do come in the door." (Focus group 1)

Some nurses described that admitting teams did not share the same amount or immediacy of responsibility when IHT patients arrived. This was a source of frustration because they perceived an urgency to get a provider to the bedside, pointing out that unlike patients admitted through the emergency department, some IHT patients may have had a longer gap since they were last evaluated by a provider team:

"I usually just blow up whoever the team is and if they're not up there within the hour I usually call again 
and then call them again. I understand we're all busy, but again, the patient hasn't even had eyes laid on them so can somebody come up and see this patient?" (Interview 9)

3b. Resumption of Care. In addition to being immediately responsible for IHT patients, nurses described the expectation that they immediately resume, if not advance, the care that was provided at the transferring facility. However, their ability to perform care tasks was contingent on the admitting team's timely assignment, evaluation of the patient, and placement of orders:

"And the first question once [IHT patients] get here is 'Can I eat and can I drink?' Or like you're in a ton of pain. That really stinks. Here's some ice. That's all I got for you. Like they were giving you morphine and fentanyl over there... sorry. Our hands are tied until your doctor gets in here." (Focus group 4)

Even in critical situations where patients clinically deteriorated at the time of arrival or shortly thereafter, nurses described having to prioritize getting a provider to the bedside for orders so they could administer care:

"I have to jump through too many hoops just to get somebody to the bedside.... [in response to a decompensating patient], I'm trying to call a medical emergency team response... [while] I'm also trying to call the triage team so that they can assign him a doctor because I have no doctor to give me orders or do anything." (Interview 5)

Nurses described that this inability to perform care tasks until provider orders were placed created significant tension with IHT patients. This tension impacted patients' perception of their quality of care and created nursing stress and professional dissatisfaction:

"[You feel] incompetent and just totally flustered and you're not being a good nurse and it's like you're not getting off to a good start with these people that have already had a rough day. And then you're just like 'I'm sorry, I'm still waiting.' It just makes me feel ... like such a baby and you're like I can't do anything. I have to wait for the doctor, and it just makes you feel not great." (Interview 17)

Participants identified solutions like improved timeliness of admitting team evaluation of IHT patients and prioritized input of orders to streamline care delivery:

"[If] the physician can come see the patient and put in an order sooner... we can act on those orders and have a better relationship with the patient - like give them pain medication and give them antibiotics that they need on time, so it just helps with the continuity of care." (Interview 2)

\section{DISCUSSION}

In this study of inpatient floor-level nurses who care for patients transferred from other facilities, our primary goal was to identify challenges and solutions to IHT care coordination to guide future interventions. Participants described an absence of standardized processes and communication practices to coordinate IHT care. Unreliable information exchange and inefficient communication structures lead to incomplete delivery of key clinical information. As a result, nurses expressed difficulty anticipating and preparing for IHT patient needs. Additionally, delays in admitting provider team evaluation of the patient and order placement resulted in patient care delays, created tension between nurses and patients, and contributed to nursing stress and professional dissatisfaction. The COVID-19 pandemic, which occurred halfway through our study, did not create additional issues for nurses, but highlighted many of the same issues nurses experienced with IHTs prior to COVID-19. The findings of this study can be leveraged as key targets for future IHT improvement efforts.

Prior work in studying IHT processes identified inadequate communication practices between physicians on the care team and physicians and patients. ${ }^{2,11,13}$ These included varied communication practices among transferring, accepting, and admitting physicians, inconsistent or incomplete receipt of relevant clinical information, and disorganization of records. ${ }^{11-13}$ Shared electronic health records and clear communication chains were seen as possible solutions to help with information exchange fidelity. ${ }^{11-13}$ Our work demonstrates that these communication challenges extend to other key stakeholders in IHT care coordination, specifically floor-level bedside nurses who are responsible for preparing for the patient's arrival, coordinating with admitting teams, and delivering care.

Additionally, this study offers a structured lens through which to characterize and understand the IHT process by employing the AHRQ Care Coordination Measurement Framework. From a nursing perspective, ideal IHT care coordination depends on complete and timely transmission of relevant clinical information, bidirectional communication structures, shared understanding of the care plan, and clear roles and responsibilities, all key components to the AHRQ framework. Our participants offered solutions that complement and expand upon each of these components, like standardization and bidirectionality to improve communication, clearer team assignments and greater involvement of nurses to enhance assessment of patient needs and goals, and timely provider evaluation and orders to expedite care delivery. In other care transition studies, intra-hospital team handoff standardization has been shown to reduce medical errors. ${ }^{31-34}$ 
Accepting institutions could consider including a checklist of equipment, personnel, and safety items to ensure appropriate nursing preparation. Furthermore, creation of an IHT team huddle at the bedside for nurses and admitting providers could facilitate a shared mental model of the patient, address immediate patient needs, and establish a clear treatment plan.

Our work also highlights the unequal responsibility that bedside nurses assume in initial IHT care at accepting facilities. Beyond being physically responsible for the patient, nurses are also accountable to patients for knowing their care plan, managing their needs, and ensuring their safety. These implicit nursing responsibilities have been documented in the nursing literature ${ }^{35,36}$ and are likely more pronounced given the assumed clinical stability of floor-level IHT patients by admitting teams. Additionally, floor-level patients, unlike critically ill patients, are often conversant and engaged in their care. As our results demonstrate, incomplete information and limited autonomy in IHT impede nurses' ability to fulfill their care responsibilities. This forces nurses into a primarily reactive care model instead of a proactive care model and creates unnecessary stress. Nurses described feeling powerless to deliver timely and appropriate care for IHT patients while they waited for the admitting team's assessment and orders. Recognition of the importance of timely evaluation and prioritization of IHT patients by admitting teams can ameliorate these stressors and better acknowledge and correct the disparity in responsibilities that nurses hold in IHT. Other potential solutions include basic standing orders and assignment of admitting teams ahead of patient arrival with contact information readily available.

Limitations to this work include a mostly White sample of nurses who primarily worked day shifts, which may not capture the full range of perspectives despite our efforts to recruit equally across day and night shifts. There may have been selection bias since nurses may have opted to participate to discuss negative experiences. We attempted to mitigate this by asking about both positive and negative IHT experiences equally. Additionally, perspectives from other groups (e.g., physicians, patients) involved in IHT care are not included. Our results may not be generalizable beyond the context of the study participants at this single center, as IHT protocols are different across institutions and service lines. However, challenges in the IHT process described in this study are consistent with problematic areas identified in prior limited research, suggesting similar experiences at other institutions. ${ }^{2,11,13} \mathrm{Al}$ though our interviews and focus groups were limited to inpatient floor-level nurses at one hospital site, we sampled a large quaternary hospital with similar numbers of annual IHT patients as other studies, ${ }^{13}$ nurses across different geographic units that may have different approaches to caring for adult medicine patients, and over a large length of time. In addition, $81 \%$ of the nurses who participated were female, which is comparable to the $90 \%$ of nurses in the industry that are female. $^{37}$
The findings in this study suggest that development of standardized nursing handoff reports, timely clinician evaluation of patients and input of orders, and improved communication models could help floor-level bedside nurses better care for IHT patients. Targeting these issues are particularly important as nurses are often the first contact for IHT patients, who look to nurses to provide them information and administer care. Additionally, physicians rely on nurses to bridge the time between patient arrival and their evaluation, creating a space where nurses are physically and clinically responsible for the patient, but are also lacking authority and knowledge. By addressing these issues, patient satisfaction, patient safety, and nursing professional satisfaction could be potentially improved. Future work should study other perspectives of those involved in the IHT process, including other healthcare team members as well as patients and their families, and the interdependent nature of IHT care. Studying these populations and triangulating their perspectives with nursing perspectives in this study could inform development of standardized processes, targetable quality metrics, and eventually, national guidelines for IHT.

Supplementary Information The online version contains supplementary material available at https://doi.org/10.1007/s11606-02107276-5.

Acknowledgements: We are grateful to the study participants for sharing their experiences and to Dr. Jacinda Nicklas and Dr. Andrea Ma for their insightful comments.

Corresponding Author: Amy Yu, MD; Division of Hospital Medicine, Department of Medicine, University of Colorado Anschutz Medical Campus, 12401 E. 17th Avenue Mailstop F-782, Aurora, CO 80045, USA (e-mail: amy.yu@cuanschutz.edu).

Funding This work was supported by Grant 2015212 from the Doris Duke Charitable Foundation and the Division of Hospital Medicine at the University of Colorado School of Medicine.

Dr. Gilmartin is supported by Career Development Award Number 1IK2HX002587-01A1 from the United States Department of Veterans Affairs Health Services Research \& Development Service of the VA Office of Research and Development.

Dr. Mueller is funded by 5KO8HS023331 from the Agency for Healthcare Research and Quality (AHRQ).

Dr. Jones is funded by K08HSO24569 from the Agency for Healthcare Research and Buality (AHRQ).

The content of this manuscript is solely the responsibility of the authors and does not necessarily represent the official views of the Doris Duke Charitable Foundation, the University of Colorado, or AHRQ.

Data Availability The datasets used and/or analyzed during the current study are available from the corresponding author on reasonable request.

\section{Declarations:}

Conflict of Interest: The authors declare that they do not have a conflict of interest.

Disclosures: The authors have no disclosures to declare. 


\section{REFERENCES}

1. Hernandez-Boussard T, Davies S, McDonald K, Wang NE. Interhospital Facility Transfers in the United States: A Nationwide Outcomes Study. J Patient Saf. 2017;13(4):187-191.

2. Bosk EA, Veinot T, Iwashyna TJ. Which patients and where: a qualitative study of patient transfers from community hospitals. Med Care. 2011;49(6):592-598. https://doi.org/10.1097/MLR.0b013e31820fb71b.

3. Coleman EA, Min SJ, Chomiak A, Kramer AM. Posthospital care transitions: patterns, complications, and risk identification. Health Serv Res. 2004;39:1449-1465.

4. Dy SM, Rubin HR, Lehmann HP. Why do patients and families request transfers to tertiary care? A qualitative study. Soc Sci Med 2005;61(8): 1846-4.

5. Wagner J, Iwashyna TJ, Kahn JM. Reasons underlying interhospital transfers to an academic medical intensive care unit. J Crit Care. 2013;2 (2):202-208. https://doi.org/10.1016/j.jcrc.2012.07.027.

6. Pakula AM, Gannon J, Mundy L, Berns K, Stoltenberg A, Ballinger, BA. (2016) Inter-hospital Critical Care Transport: Implementation of a Novel Policy and Review of the Literature. Int $J$ Crit Care Emerg Med 2:011. https://doi.org/10.23937/2474-3674/1510011.

7. Steg PG, James SK, Atar D, et al. ESC Guidelines for the management of acute myocardial infarction inpatients presenting with ST segment elevation. Eur Heart J 2012;33:2569-619.

8. Widimsky P, Bilkova D, Penicka M, et al. Long-term outcomes of patients with acute myocardial infarction presenting to hospitals without catheterization laboratory and randomized to immediate thrombolysis or interhospital transport for primary percutaneous coronary intervention. Five years' follow-up of the PRAGUE-2 Trial. Eur Heart J 2007;28:679 84.

9. Aguirre FV, Varghese JJ, Kelley MP, et al. Rural interhospital transfer of ST-elevation myocardial infarction patients for percutaneous coronary revascularization: the Stat Heart Program. Circulation 2008;117:114552.

10. McDonald KM, Schultz E, Albin L, et al. Care Coordination Atlas Version 4 (Prepared by Stanford University under subcontract to American Institutes for Research on Contract No. HHSA290-2010-00005I). AHRQ Publication No. 14-0037- EF. Rockville, MD: Agency for Healthcare Research and Quality. June 2014.

11. Mueller SK, Shannon E, Dalal A, Schnipper JL, Dykes P. Patient and Physician Experience with Interhospital Transfer: A Qualitative Study. J Patient Saf. 2018. https://doi.org/10.1097/PTS.0000000000000501.

12. Finn EB, Campbell Britton MJ, Rosenberg AP, et al. A Qualitative Study of Risks Related to Interhospital Transfer of Patients with Nontraumatic Intracranial Hemorrhage. J Stroke Cerebrovasc Dis. 2019;28(6):17591766.

13. Herrigel DJ, Carroll M, Fanning C, Steinberg MB, Parikh A, Usher M. Interhospital transfer handoff practices among US tertiary care centers: A descriptive survey. J Hosp Med. 2016;11(6):413-417.

14. Kripalani S, LeFevre F, Phillips CO, Williams MV, Basaviah P, Baker DW. Deficits in Communication and Information Transfer Between HospitalBased and Primary Care Physicians: Implications for Patient Safety and Continuity of Care. JAMA. 2007;297(8):831-841. https://doi.org/10. 1001/jama.297.8.831.

15. Moore C, Wisnivesky J, Williams S, McGinn T. Medical errors related to discontinuity of care from an inpatient to an outpatient setting. J Gen Intern Med. 2003;18(8):646-651. https://doi.org/10.1046/j.1525-1497. 2003.20722.x.

16. Bodenheimer T. Coordinating care-a perilous journey through the health care system. N Engl J Med 2008;358:1064 -71.

17. Rattray NA, Sico JJ, Cox LM, Russ AL, Matthias MS, Frankel RM. Crossing the Communication Chasm: Challenges and Opportunities in Transitions of Care from the Hospital to the Primary Care Clinic. Jt Comm J Qual Patient Saf. 2017 Mar;43(3):127-137. https://doi.org/10. 1016/j.jcjq.2016.11.007. Epub 2016 Nov 15.
18. Shrank WH, Rogstad TL, Parekh N. Waste in the US Health Care System: Estimated Costs and Potential for Savings. JAMA. 2019;322(15):15011509. https://doi.org/10.1001/jama.2019.13978.

19. Mohr NM, Wong TS, Faine B, Schlichting A, Noack J, Ahmed A. Discordance Between Patient and Clinician Experiences and Priorities in Rural Interhospital Transfer: A Mixed Methods Study. J Rural Health. 2016;32(1):25-34.

20. Gustafsson M, Wennerholm S, Fridlund B. Worries and concerns experienced by nurse specialists during inter-hospital transports of critically ill patients: a critical incident study. Intensive Crit Care Nurs. 2010;26(3):138-145

21. Eiding H, Kongsgaard UE, Braarud AC. Interhospital transport of critically ill patients: experiences and challenges, a qualitative study. Scand J Trauma Resusc Emerg Med. 2019;27(1):27.

22. Patton MQ. Enhancing the quality and credibility of qualitative analysis. Health Serv Res. 1999;34(5 Pt 2):1189-1208.

23. Trotter RT, 2nd. Qualitative research sample design and sample size: resolving and unresolved issues and inferential imperatives. Prev Med. 2012;55(5):398-400.

24. Coyne IT. Sampling in qualitative research. Purposeful and theoretical sampling; merging or clear boundaries? J Adv Nurs. 1997;26(3):623-630.

25. Tong A, Sainsbury P, Craig J. Consolidated criteria for reporting qualitative research (COREQ): a 32-item checklist for interviews and focus groups. Int J Qual Health Care. 2007;19(6):349-357.

26. Morse JM. Critical Analysis of Strategies for Determining Rigor in Qualitative Inquiry. Qual Health Res. 2015;25(9):1212-1222.

27. Kuper A, Lingard L, Levinson W. Critically appraising qualitative research. BMJ. 2008;337:a 1035.

28. Saunders B, Sim J, Kingstone T, et al. Saturation in qualitative research: exploring its conceptualization and operationalization. Qual Quant. 2018;52(4): 1893-1907.

29. Creswell J. Qualitative Inquiry and Research Design: Choosing Among Five Approaches. 3rd Edition. Thousand Oaks, CA: SAGE Publications, Inc; 2012.

30. Fereday J, Muir-Cochrane E. Demonstrating Rigor Using Thematic Analysis: A Hybrid Approach of Inductive and Deductive Coding and Theme Development. Int J Qual Methods. 2006;5(1):80-92.

31. Starmer AJ, Sectish TC, Simon DW, et al. Rates of medical errors and preventable adverse events among hospitalized children following implementation of a resident handoff bundle. JAMA. 2013;310(21):2262-2270.

32. Joy BF, Elliott E, Hardy C, Sullivan C, Backer CL, Kane JM. Standardized multidisciplinary protocol improves handover of cardiac surgery patients to the intensive care unit. Pediatr Crit Care Med. 2011;12(3):304-308.

33. Malpass HC, Enfield KB, Keim-Malpass J, Verghese GM. The Interhospital Medical Intensive Care Unit Transfer Instrument Facilitates Early Implementation of Critical Therapies and Is Associated With Fewer Emergent Procedures Upon Arrival. J Intensive Care Med. 2015;30(6):351-357.

34. Theobald CN, Choma NN, Ehrenfeld JM, Russ S, Kripalani S. Effect of a Handover Tool on Efficiency of Care and Mortality for Interhospital Transfers. J Hosp Med. 2017;12(1):23-28.

35. Hughes RG. Nurses at the "Sharp End" of Patient Care. In: Hughes RG, editor. Patient Safety and Quality: An Evidence-Based Handbook for Nurses. Rockville (MD): Agency for Healthcare Research and Quality (US); 2008 Apr. Chapter 2. Available from: https://www.ncbi.nlm.nih.gov/ books/NBK2672/.

36. Medicine Io. Keeping Patients Safe: Transforming the Work Environment of Nurses. Washington, DC: The National Academies Press; 2004:31-49.

37. Smiley RA, Lauer P, Bienemy C, et al. The 2017 National Nursing Workforce Survey. J Nurs Regul. 2018;9(3) S1-S88.

Publisher's Note: Springer Nature remains neutral with regard to jurisdictional claims in published maps and institutional affiliations. 